Article

\title{
Morphological Transitions of Photoresponsive Vesicles from Amphiphilic Polypeptoid Copolymers for Controlled Release
}

\author{
Xu Yang, Zhiwei Wang and Jing Sun *(1) \\ Key Laboratory of Biobased Polymer Materials, Shandong Provincial Education Department, School of Polymer \\ Science and Engineering, Qingdao University of Science and Technology, Qingdao 266042, China; \\ qust_yangxu@163.com (X.Y.); polymerwzw@foxmail.com (Z.W.) \\ * Correspondence: jingsun@qust.edu.cn
}

Received: 9 March 2020; Accepted: 26 March 2020; Published: 3 April 2020

\begin{abstract}
Photoresponsive polymers have attracted increasing interest for a variety of applications. Here, we report a family of photoresponsive polypeptoid-based copolymer poly(ethylene glycol)-b-poly(N-(S-(o-nitrobenzyl)-thioethyl) glycine)-co-poly(N-(2-phenylethyl) glycine) (PEG-b-PNSNco-PNPE) synthesized by the controlled ring-opening polymerization (ROP) technique. The key feature of the design is to incorporate both $o$-nitrobenzyl group moiety to offer the photoresponsive property and phenethyl residues to tune the structural and amphiphilic property of the system. We demonstrate that the cleavage degree of the o-nitrobenzyl group can reach to $100 \%$ upon UV-irradiation. With delicate design, a photoresponsive vesicle-to-sphere transition has been observed that facilitates the release of the encapsulants. This work provides a facile approach to prepare a type of photoresponsive polymers with tunable properties for drug delivery.
\end{abstract}

Keywords: photoresponsive; polypeptoid; self-assembly; drug delivery

\section{Introduction}

Self-assemblies prepared from natural polymers and bioinspired synthetic polymers have been largely used for pharmaceutical applications due to their excellent biocompatibility and non-immunogenicity [1-8]. Stimuli-responsive self-assembly is of particular interest for versatile drug delivery as it can rapidly respond to the external stimuli in a controlled manner. Photoresponsive assemblies have attracted great attention in various fields due to the unique properties of their photoreactions, such as high electivity and efficiency. The $o$-nitrobenzyl group, as one of the photoliable groups, shows excellent stability in extreme conditions with tunable photochemical properties [1,9-13]. Photo cleavage of polymers containing o-nitrobenzyl group can generate amine, carboxylic acid, and thiol groups with high reactivity [2,14-19]. Consequently, the physicochemical properties of the assemblies largely vary that result in morphology transition or disassembly and a further release of the encapsulated payloads. The photo-responsive self-assemblies are considered as excellent tools for smart drug delivery.

Polypeptoids, also known as nitrogen-substituted polyglycines (N-polyglycine), are a class of bioinspired polymer materials with biological activity. The properties of the polypeptoids are mainly determined by the side chain moiety because of the absence of hydrogen bonding and chirality in the backbone [20-27]. Both structural and property's tunablity enables the polypeptoid-based system highly designable. We have previously prepared a type of photoresponsive polypeptoid-based diblock copolymers that can self-assemble into various morphologies. After UV-irradiation, more than one-fourth of the $o$-nitrobenzyl group and the nanostructures remain due to the cross-linking of the 
disulfide bonds. To benefit for the smart drug delivery application, in this study we synthesized a class of photoresponsive block copolymer poly(ethylene glycol)-b-poly(N-(S-(o-nitrobenzyl)-thioethyl) glycine)-co-poly(N-(2-phenylethyl) glycine) (PEG- $b$-PNSN-co-PNPE) by statistical copolymerization of a mixture of $\mathrm{N}-(\mathrm{S}-(o-n i t r o b e n z y l)-t h i o e t h y l)$ and $\mathrm{N}$-phenethyl $\mathrm{N}$-carboxyanhydrides (NCAs) using amino-terminated poly(ethylene glycol) $\left(\mathrm{mPEG}-\mathrm{NH}_{2}\right)$ as the macroinitiator by ring-opening polymerization (ROP). The advantage of the design is to incorporate both $o$-nitrobenzyl group moiety to offer the photoresponsive property and phenethyl residues to tune the structural and amphiphilic property of the system. We demonstrated that the $o$-nitrobenzyl (NB) group can be entirely cleaved to induce the free thiol groups as the irradiation time increases up to $10 \mathrm{~h}$. Simultaneously, the self-assembled morphology transforms from a few hundred nanometers of vesicles to tens of nanometers of nanospheres. This transition further results in the release of the small molecule in a controlled manner. The synthesis, self-assembly and phototriggered drug release have been thoroughly investigated.

\section{Materials and Methods}

\subsection{Materials and Instruments}

Hexane, tetrahydrofuran (THF), and dichloromethane (DCM) were purified by purging with dry nitrogen, followed by passing through columns of activated alumina. N,N-Dimethylformamide (DMF) was treated with free amine scavenger (Aldrich, Berlin, Germany) before passing through $4 \AA$ molecular sieves and activated alumina column. Ethyl acetate (EtOAc) was freshly distilled from $\mathrm{CaH}_{2}$. Methoxypolyethylene glycol amine (mPEG- $\mathrm{NH}_{2}, \mathrm{Mw}=2000 \mathrm{~g} / \mathrm{mol}$ ) was purchased from Jenkem Technology Co, Ltd. (Beijing, China). o-nitrobenzyl bromide was purchased from Ark Pharm reagent Co., Ltd. (Chicago, IL, USA). Cysteamine hydrochloride (98\%) was purchased from Admas Reagent Co., Ltd. (Shanghai, China). Phenylethylamine, di-tert-butyl dicarbonate, rhodamine B and glyoxylic acid monohydrate were purchased from Titan reagent (Shanghai, China). All other chemicals were purchased from commercial suppliers and used without further purification unless otherwise noted.

${ }^{1} \mathrm{H}$ NMR spectra were recorded on Bruker AV400 FT-NMR spectrometer. Gel permeation chromatography/laser light scattering (GPC/LLS) was performed at $50{ }^{\circ} \mathrm{C}$ using an SSI (Series I) pump (LC20AT, Shimadzu Corporation, Kyoto, Japan) connected to Wyatt Optilab DSP with $0.02 \mathrm{M}$ $\mathrm{LiBr}$ in DMF as the eluent at a flow rate of $1.0 \mathrm{~mL} / \mathrm{min}$. Conventional calibrations were performed using polystyrene standards (PS). The concentrations of all samples were about $5 \mathrm{mg} / \mathrm{mL}$. Differential scanning calorimetry (DSC) studies were conducted using a TA instrument DSC 25 (New Castle, DE, USA). Powder samples enclosed in the aluminum pans were heated from -50 to $200{ }^{\circ} \mathrm{C}$ at $10{ }^{\circ} \mathrm{C} \mathrm{min}-1$ for 3 cycles. Transmission electron microscopy (TEM) samples were examined with a JEM2200FS TEM (200 keV, Tokyo, Japan). TEM samples were prepared by pipetting polymer solution on carbon coated TEM grids and the excess amount of solution was blotted with a piece of filter paper. The hydrodynamic diameter $\left(D_{\mathrm{h}}\right)$ of the assemblies was recorded at $25^{\circ} \mathrm{C}$ by dynamic light scattering (DLS) using a Brookhaven NanoBrook instrument (Holtsville, NY, USA). The UV-vis spectra of the samples were recorded at room temperature using a Shimadzu UV-2910 spectrometer. The sample was irradiated using a mercury high pressure UV lamp (250 W, GGZ250, Shanghai Jiguang Special Lighting Electrical Factory, Shanghai, China). The samples were stored avoiding exposure from nature light.

\subsection{Synthesis of Poly(ethylene glycol)-b-poly(N-(S-(o-nitrobenzyl)-thioethyl) glycine)-co-poly(N-(2-phenylethyl) glycine) (PEG-b-PNSN-co-PNPE) Copolymer}

The synthesis of N-(S-(o-nitrobenzyl)-thioethyl) and N-phenylethyl N-carboxyanhydrides monomers has been reported in previous work $[28,29]$. $\mathrm{mPEG}-\mathrm{NH}_{2}$ was completely dried under vacuum for $30 \mathrm{~h}$ and dissolved in anhydrous THF in a polymerization tube. The solution of NSN-NCA and NPE-NCA mixtures (100 mg/mL in THF) at the given ratio was then added to the polymerization tube. The polymerization was performed under a $\mathrm{N}_{2}$ atmosphere at $55{ }^{\circ} \mathrm{C}$ and monitored by FTIR 
spectrum until the characteristic peaks $\left(1850\right.$ and $\left.1790 \mathrm{~cm}^{-1}\right)$ of NCAs disappeared. The final solution was precipitated into excess cold diethyl ether. The pale yellow oil was collected by centrifugation, washed another three times by cold diethyl ether and dried under reduced pressure to yield white to pale yellow oil (56\% yield). ${ }^{1} \mathrm{H}$ NMR $(400 \mathrm{MHz}, \mathrm{DMSO}) \delta: 3.53-2.97(\mathrm{t}, 70 \mathrm{H}), 3.23(\mathrm{~s}, 3 \mathrm{H}), 3.05-3.44(\mathrm{t}$, $70 \mathrm{H}), 3.50(\mathrm{~s}, 176 \mathrm{H}), 3.76-4.70(\mathrm{~s}, 92 \mathrm{H}), 6.87-7.35(\mathrm{~m}, 120 \mathrm{H}), 7.36-7.71(\mathrm{~m}, 33 \mathrm{H})$, and $7.85-8.05(\mathrm{~d}, 11 \mathrm{H})$.

In the entire procedure, the polymer was avoided exposure to nature light.

\subsection{Cleavage of Copolymers with UV Irradiation}

Sixty milligrams of PEG-b-PNSN-co-PNPE copolymer was dissolved in $6 \mathrm{~mL}$ of anhydrous DCM in a glass vial and 0.01 equivalent of dimethylphenylphosphine (DMPP) was added. Afterwards, the solution was exposed to a mercury high pressure UV lamp. $1 \mathrm{~mL}$ of the solution was taken at intervals of 2, 4, 6, 8, and $10 \mathrm{~h}$, respectively, and precipitated with an excess of diethyl ether for three times. The product was dried under vacuum at $30^{\circ} \mathrm{C}$ before measurement.

\subsection{Preparation of Assemblies in Aqueous Solution}

The PEG- $b$-PNSN-co-PNPE copolymer $(10 \mathrm{mg}$ ) was first dissolved in $1 \mathrm{~mL}$ of THF, then $20 \mathrm{~mL}$ of deionized water was slowly added dropwise with stirring. After the addition of water was completed, the solution was stirred at room temperature for another $48 \mathrm{~h}$. Then the THF was removed by a rotary evaporator. The solution was stirred under a mercury high pressure UV lamp for $10 \mathrm{~h}$.

\subsection{Preparation of Rhodamine B Loaded Vesicles in Aqueous Solution}

Ten milligrams of PEG- $b$-PNSN-co-PNPE was dissolved in $1 \mathrm{~mL}$ of THF, and then $5 \mathrm{mg}$ of rhodamine $B$ in aqueous solution $(20 \mathrm{~mL})$ was slowly dropped into the polymer solution with stirring. The solution was stirred for another $48 \mathrm{~h}$ at room temperature and then dialyzed (MWCO $=3500$, molecular weight cutoff) against deionized water to remove unloaded rhodamine B. The solution outside the dialysis bag was measured by a UV-vis spectrometer at $553 \mathrm{~nm}$ and the weight of the loaded drug was then obtained.

\subsection{In Vitro Drug Release Profiles}

Five milliliters of the rhodamine B-loaded vesicles solution $(0.5 \mathrm{mg} / \mathrm{mL})$ was placed in a dialysis bag $(\mathrm{MWCO}=3500)$ and dialyzed against $25 \mathrm{~mL}$ of PBS buffer at $37^{\circ} \mathrm{C}$ with continuous shaking. The dialysis solution was periodically replaced, and the value at $553 \mathrm{~nm}$ was recorded by a UV-vis spectrometer and the weight of the released drug was calculated. For the UV-irradiated sample, the same procedure was performed except that the solution was first irradiated under the UV lamp for $6 \mathrm{~h}$.

\section{Results and Discussion}

The N-(S-(o-nitrobenzyl)-thioethyl)-N-carboxyanhydride (NSN-NCA) and N-phenylethyl N-carboxyanhydride (NPE-NCA) monomers were synthesized as reported elsewhere (Scheme S1). The chemical structure of the monomers was confirmed by ${ }^{1} \mathrm{H}$ NMR spectroscopy (Figure 1a,b, Figures S1 and S2). The copolymers (PEG- $b$-PNSN-co-PNPE) were then synthesized by statistical copolymerization of a mixture of NSN-NCA and NPE-NCA using mPEG-NH ${ }_{2}\left(\mathrm{M}_{n}=2000 \mathrm{~g} / \mathrm{mol}\right)$ as the macroinitiator (Scheme 1). The polymerization process was monitored by FTIR. The disappearance of two characteristic $v_{\mathrm{C}=\mathrm{O}}$ peaks of the monomer at $1780 \mathrm{~cm}^{-1}$ and $1850 \mathrm{~cm}^{-1}$ was considered as complete conversion of NCA monomers to polypeptoids. The ${ }^{1} \mathrm{H}$ NMR spectra show that all peaks of the synthesized copolymers are well assigned, confirming their chemical structures (Figure 1c and Figure S3). A series of copolymers were synthesized by varying the ratio of NSN-NCA to NPE-NCA and monomers to PEG-NH $\mathrm{NH}_{2}$ macroinitiator. The average DP (degree of polymerization) of PNSN was held fixed at approximately 10 and 19, and the average DP of PNPE was varied from 10 to 34 . All the molecular characteristics of the copolymers PEG- $b-\mathrm{PNSN}_{\mathrm{m}}-\mathrm{co}-\mathrm{PNPE}_{\mathrm{n}}$ are shown in Table 1 . The GPC 
traces (Figure S4) show unimodal peaks and narrow molecular weight distribution with dispersities $(\nexists)<1.25$ for all the diblock copolymers (Table 1). This indicates that the statistical copolymerization of PNSN and PNPE monomers is well-controlled. We investigated the thermal properties of the samples by DSC (Figure S5a). The samples with and without irradiation show the absence of crystallization and melting peaks in the entire experimental window by DSC (Figure S5). As previously reported [28], the presence of the PNSN block significantly inhibits the crystallization properties of the PEG block and the PNPE block.

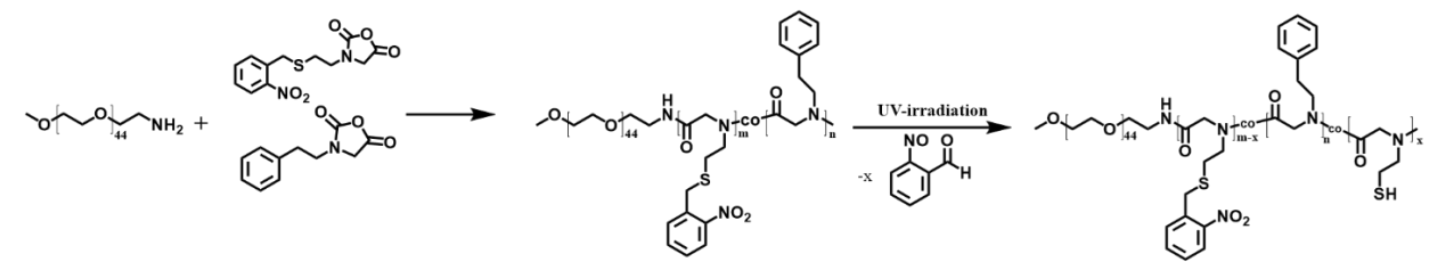<smiles>COCCOCCNC(=O)CN(CCSCc1ccccc1[N+](=O)[O-])OC(=O)CN(C)CCc1ccccc1</smiles>

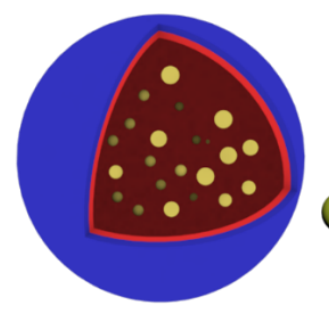

UV-irradiation

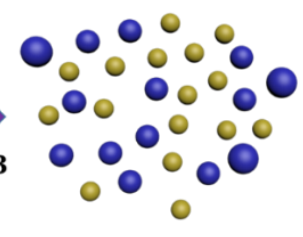

Scheme 1. The phototriggered assemblies from the poly(ethylene glycol)-b-poly(N-(S-(o-nitrobenzyl)thioethyl) glycine)-co-poly(N-(2-phenylethyl) glycine) (PEG-b-PNSN-co-PNPE) triblock random copolymer.

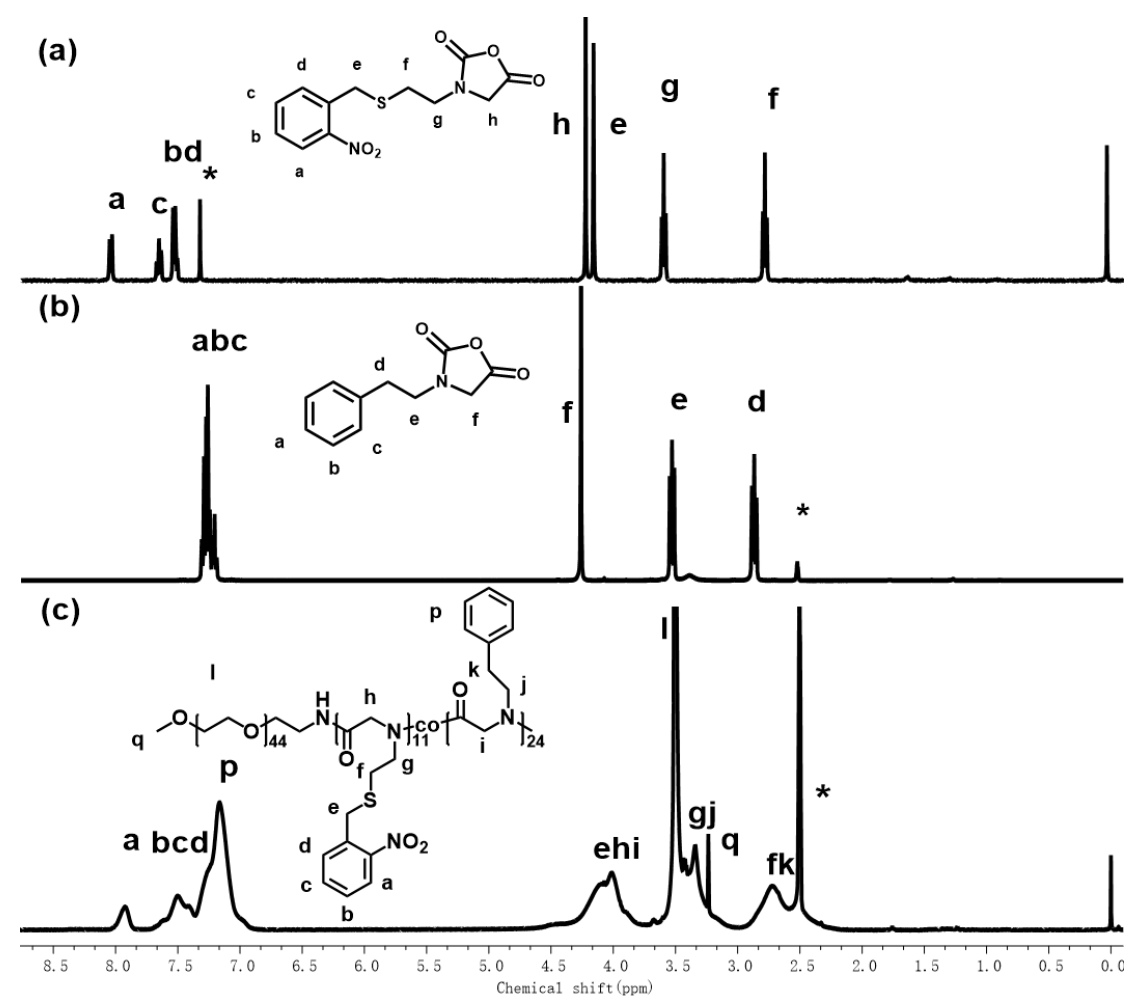

Figure 1. ${ }^{1} \mathrm{H}$ NMR spectra of (a) NSN-NCA in $\mathrm{CDCl}_{3}$, (b) NPE-NCA and (c) PEG-b-PNSN-co-PNPE in DMSO. * indicates solvents. 
Table 1. Characteristics of the triblock random copolymers synthesized.

\begin{tabular}{|c|c|c|c|c|}
\hline Samples & Feed Ratio $^{\text {a }}$ & $M_{n}{ }^{b}(k D a)$ & $M_{n}{ }^{c}(k D a)$ & Dispersity $(\boxplus)^{c}$ \\
\hline PEG- $b$-PNSN ${ }_{8}-c o-\mathrm{PNPE}_{28}$ & $1 / 10 / 30$ & 8524 & 9833 & 1.25 \\
\hline PEG- $b$-PNSN ${ }_{9}-c o-\mathrm{PNPE}_{32}$ & $1 / 10 / 40$ & 9420 & 12,500 & 1.17 \\
\hline PEG- $b$-PNSN $11-c o-\mathrm{PNPE}_{24}$ & $1 / 10 / 20$ & 8636 & 11,410 & 1.14 \\
\hline PEG- $b$-PNSN $19-c o-\mathrm{PNPE}_{10}$ & $1 / 20 / 10$ & 8398 & 9393 & 1.18 \\
\hline
\end{tabular}

${ }^{a}$ Feed molar ratio of PEG/NSN-NCA/NPE-NCA. ${ }^{b}$ Calculated from ${ }^{1} \mathrm{H}$ NMR spectra. ${ }^{c}$ Determined from Gel permeation chromatography (GPC).

As expected, the obtained copolymers exhibit photoresponsive behavior upon UV irradiation. This is due to the presence of the o-nitrobenzyl (NB) group, which can be photocleaved to generate free thiol groups. The chemical structures of copolymers with different irradiation times were studied by ${ }^{1} \mathrm{H}$ NMR, as shown in Figure S6. The NB group is gradually eliminated from the polymers during the irradiation process, as indicated by the decreased peaks at 7.4 and $7.9 \mathrm{ppm}$. Unlike previous reports, the samples (with DP $\mathrm{PNSN}_{\mathrm{N}}$ of 10 ) remain good solubility after prolonged irradiation. This is possibly because the presence of the PNPE block increases the solubility of the system. More importantly, the PNPE may also behave as steric hindrance to reduce the number of cross-links that benefits the deprotection procedure. The ${ }^{1} \mathrm{H}$ NMR results show that $100 \% \mathrm{NB}$ groups are cleaved from the PEG- $b$-PNSN $8-c o-\mathrm{PNPE}_{28}, \mathrm{PEG}-b-\mathrm{PNSN}_{9}-c o-\mathrm{PNPE}_{34}$, and PEG- $b$-PNSN $11-c o-\mathrm{PNPE}_{24}$ copolymer after $10 \mathrm{~h}$ UV irradiation (Figure 2). In the case of PEG- $b-\mathrm{PNSN}_{19}-c 0-\mathrm{PNPE}_{10}$, merely $70 \%$ NB groups are cleaved, because of the high molar ratio of PNSN that decreases the solubility of the system.

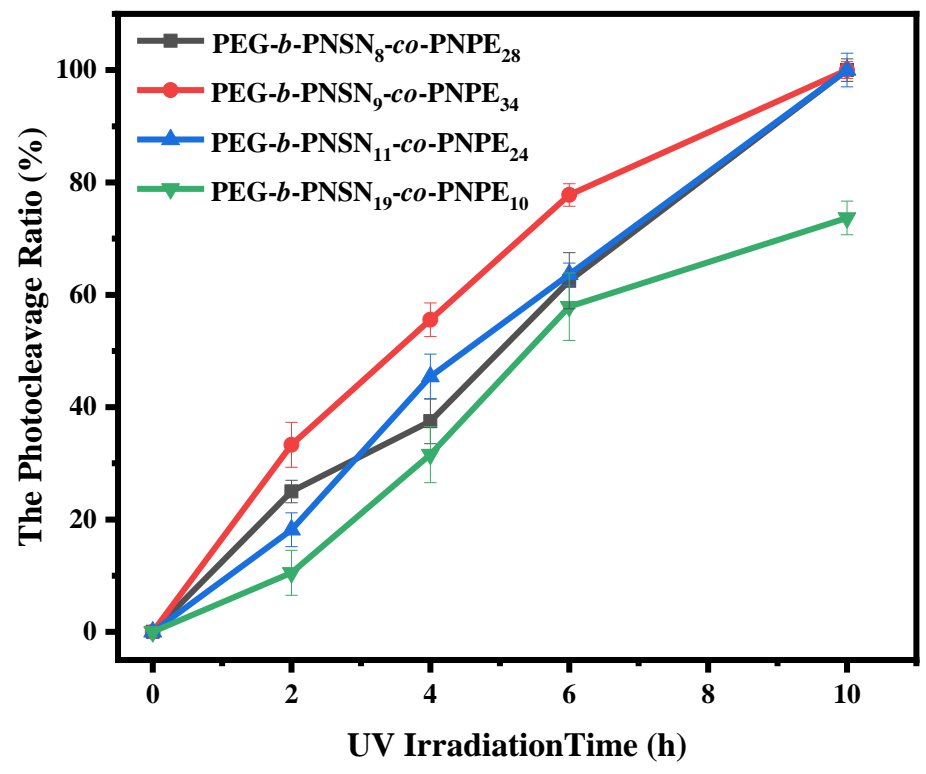

Figure 2. The photocleavage ratio of copolymers with different UV-irradiation time.

We further investigated the photoresponsive properties of PEG- $b$-PNSN-co-PNPE assemblies in aqueous solution. The copolymer was first dissolved in THF and then deionized water was added to induce molecular assembly. After removal of THF, the final concentration of all four samples was fixed at $0.5 \mathrm{mg} / \mathrm{mL}$. After exposure to UV light, the colorless assembled solution changed to yellow, which indicates that a photocleavage reaction occurs. This process is further monitored by UV-Vis spectrometer (Figure 3). With increasing the irradiation time, the enhanced characteristic absorption peak at $350 \mathrm{~nm}$ and the decreased peak at $280 \mathrm{~nm}$ indicate that the NB group is gradually photocleaved from the copolymer and produces 2-nitrosobenzaldehyde. This is coincident with previously reported results. The UV irradiation removes the hydrophobic NB group and free thiol group is generated. 
The thiol group can be further oxidized to a disulfide bond in solution, which promotes cross-linking of the components.

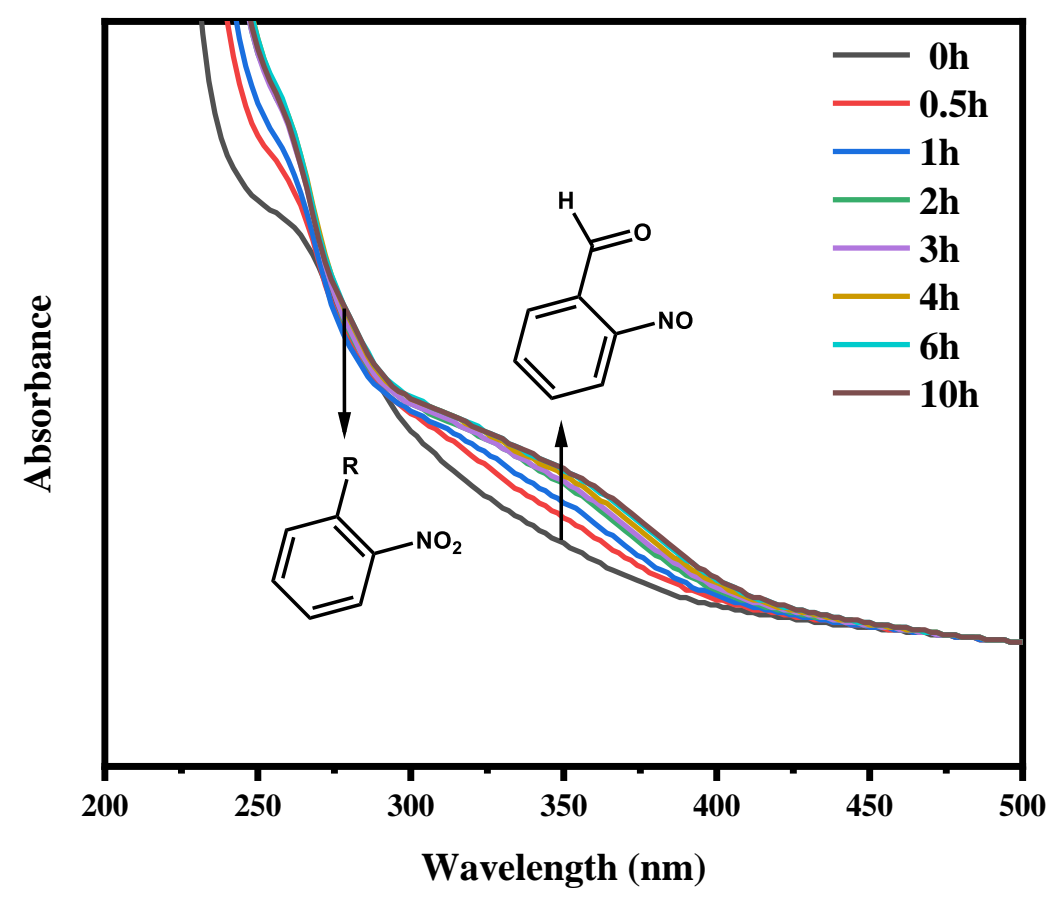

Figure 3. UV-vis spectra of PEG- $b$-PNSN $11-c o-\mathrm{PNPE}_{24}$ in aqueous solution $(0.5 \mathrm{mg} / \mathrm{mL})$ with different UV-irradiation times.

To probe the nanostructure and size of the copolymer before and after UV irradiation, TEM and DLS were performed. In all cases, the samples exhibit similar self-assembly behavior (Figure 4, Figures S7 and S8). The PEG- $b-\mathrm{PNSN}_{8}-\mathrm{co}-\mathrm{PNPE}_{28}$ without UV irradiation self-assembles into the vesicles with a diameter of $268.65 \pm 11.4 \mathrm{~nm}$, as observed by TEM (Figure 4a). The contrast between the bright center and the dark periphery suggests a vesicular hollow structure. The DLS result (Figure 4c) shows a consistent hydrodynamic diameter $\left(D_{\mathrm{h}}\right)$ of $281.57 \pm 10.6 \mathrm{~nm}$, confirming the TEM images (Figure $4 \mathrm{a}$ ). Note that water is a good solvent for PEG and non-solvent for hydrophobic PNSN and PNPE blocks. It is expected that the random PNSN-co-PNPE block forms the inner layer of the vesicle flanked by PEG out layers. As the DP of PNSN block is fixed at around 10, the size of the vesicles slightly decreases with the DP of PNPE block increasing, as indicated by the TEM images and DLS results (Figure 5). We assume that this is possibly because the increased hydrophobicity results in a reduced aggregation number for more stable nanostructures. After $10 \mathrm{~h}$ irradiation, the self-assembled morphology transforms into the spherical morphology with a diameter of $26.5 \pm 5.1 \mathrm{~nm}$ by TEM (Figure $4 \mathrm{~b}$ ). Upon exposure, the NB group is cleaved, which induces the free thiol groups. The hydrophobicity the copolymer decreases significantly, which enables the vesicle-to sphere transition. Note that the thiol groups can be further oxidized into disulfide bonds in solution in the presence of oxygen. This is very different from the results of PEG- $b$-PNSN in a previous report, where the morphology is stabilized by the disulfide bonds [28]. In this case, the random distribution of PNSN and PNPE blocks may disrupt the cross-linking of thiol groups on PNSN. Thus, the morphology transition occurs prior to cross-linking. The $D_{\mathrm{h}}$ of the spheres measured by DLS (Figure 4d) is $43.4 \pm 8.6 \mathrm{~nm}$, larger than that observed from TEM measurement (Figure $4 \mathrm{~b}$ ). This may be due to the volume shrinkage of the cross-linked assemblies during drying process and invisible corona with low electron density by TEM [3]. All the other copolymers show similar behavior (Figure S7). With fixed DP of PNSN, the size of the micelles decreases with the DP of PNPE block increasing, similar to that before UV-irradiation (Figure 5). 

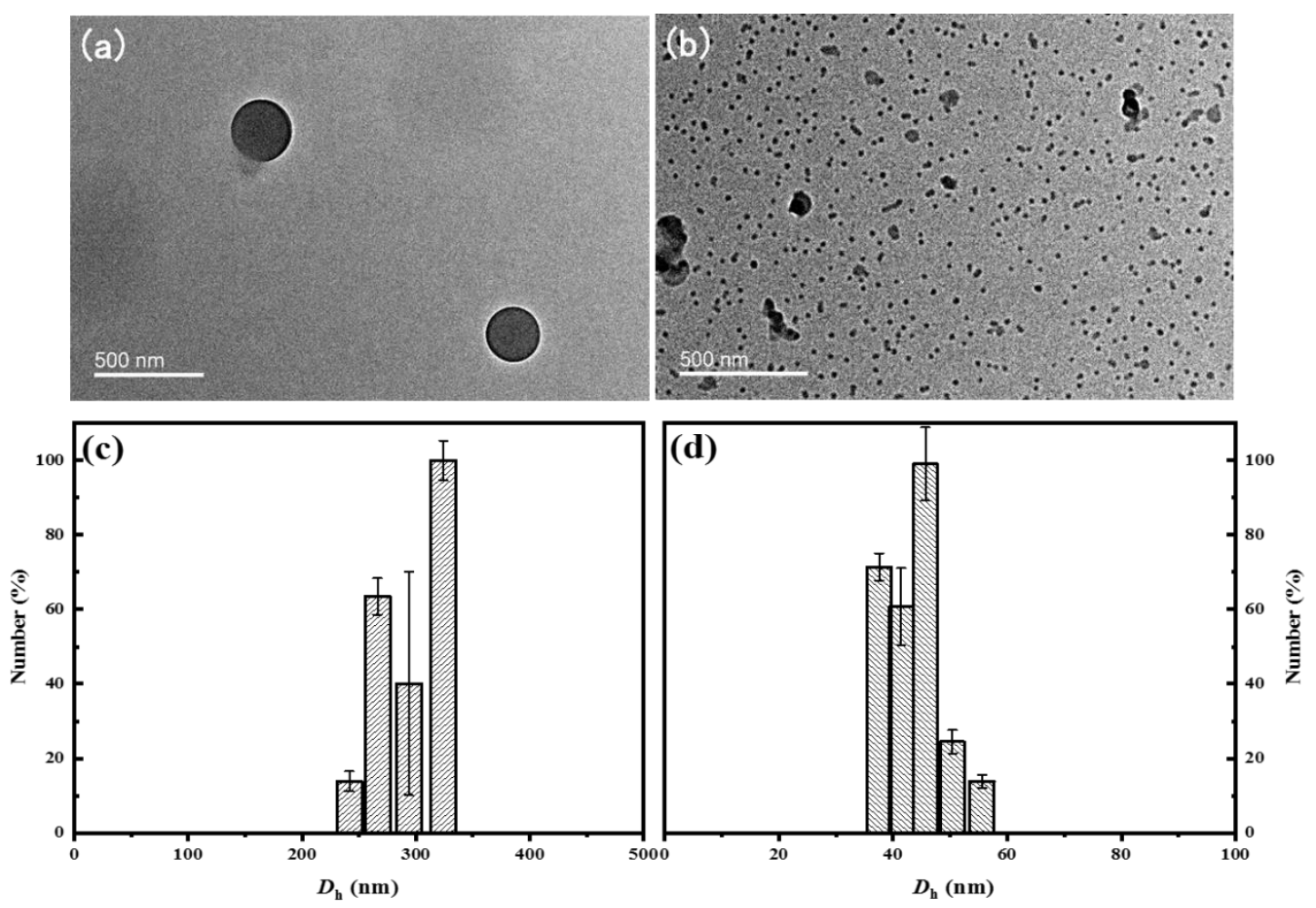

Figure 4. The Transmission electron microscopy (TEM) images of PEG- $b-\mathrm{PNSN}_{8}-\mathrm{co}-\mathrm{PNPE}_{28}$ with non-irradiation (a) and with $10 \mathrm{~h}$ irradiation (b); the $D_{\mathrm{h}}$ with non-irradiation (c), and with $10 \mathrm{~h}$ irradiation (d) in aqueous solution.

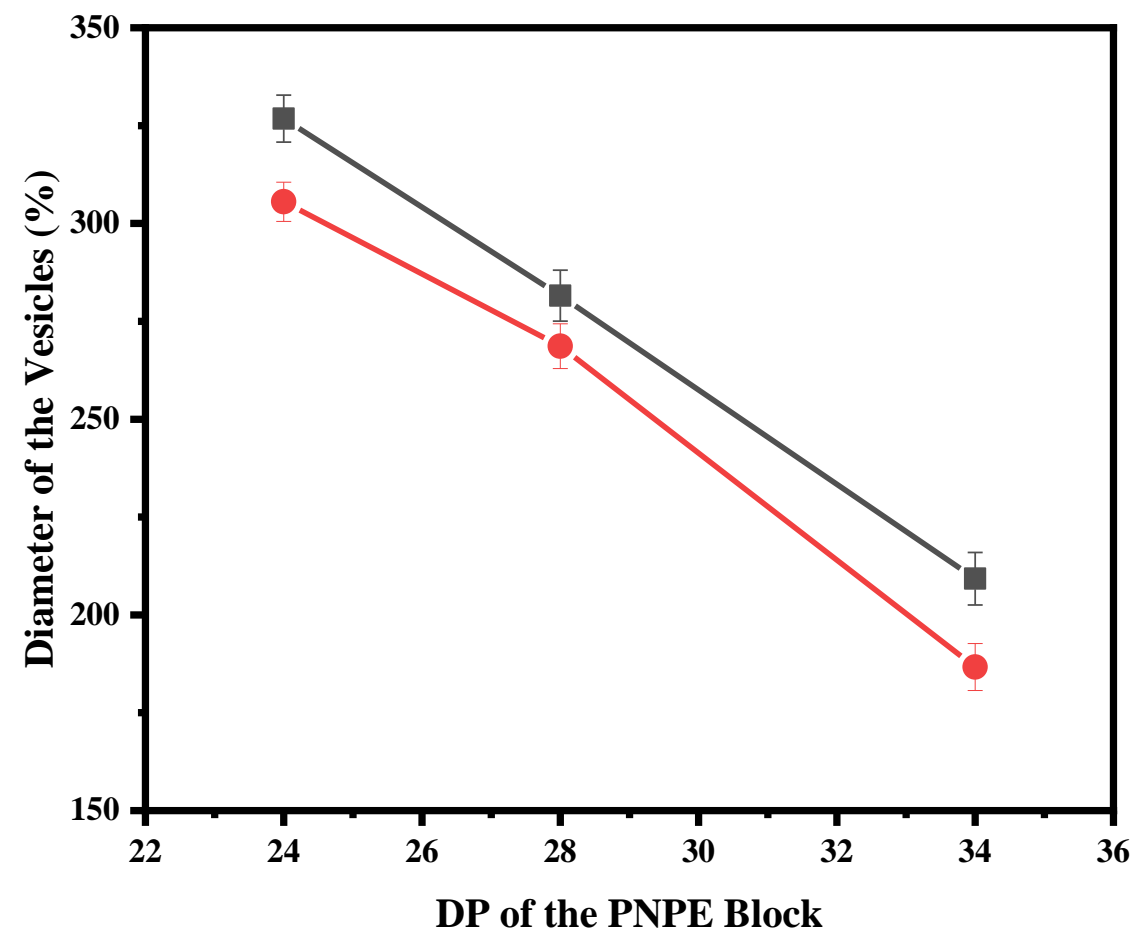

Figure 5. The relationship between DP(degree of polymerization) of the PNPE block and diameter of the vesicles as the DP of PNSN block is fixed at around 10. The red circle represents the results observed by TEM and the black square represents the results determined by dynamic light scattering (DLS). 
The dramatic morphology variation facilitates the prompt release of encapsulates. Thus, we designed and studied the drug loading and release capacity of the PEG-b-PNSN-co-PNPE carrier. Note that most of the studies focus on the encapsulation of the hydrophobic drugs such as doxorubicin [3]. Considering the aqueous interior of the vesicle, we used a hydrophilic fluorescent probe rhodamine $B$ for further study. Rhodamine B was loaded into the PEG- $b$-PNSN $19-c o-\mathrm{PNPE}_{10}$ vesicles by co-solvent assembly and subsequent dialysis, which results in the load capacity of $34.5 \mathrm{wt} . \%$ by UV-Vis spectrometer. The assembled solutions with/without irradiation were then placed in PBS $\left(37^{\circ} \mathrm{C}\right)$ simultaneously for further investigation of in vitro release profile. Figure 6 shows that both systems show similar release kinetics, where rapid release is observed in the initial stage and turns to modest increase tendency. Further, the sample after $6 \mathrm{~h}$ irradiation shows much pronounced release rate compared with the non-irradiated sample (Figure 6). Upon irradiation, the cumulative drug release reached $\sim 40 \%$ in $60 \mathrm{~h}$, which is nearly four-times than that of non-irradiated samples. This variation suggests that the hydrophilic molecules diffuse dramatically from the vesicles due to the photo-triggered morphology transition. Our system provides a new solution for the photo-responsive delivery of hydrophilic drugs for biomedical applications.

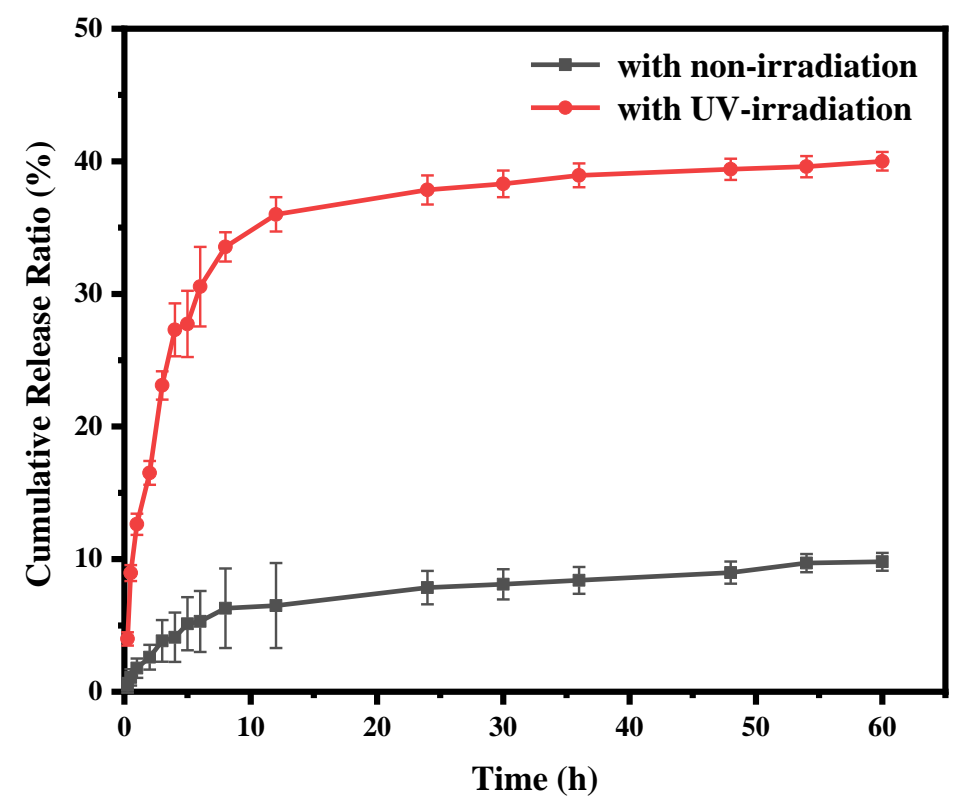

Figure 6. In vitro drug-release profiles of rhodamine B loaded PEG- $b$-PNSN $19-c o-\mathrm{PNPE}_{10}$.

\section{Conclusions}

In summary, we have successfully synthesized a family of photoresponsive polypeptoid-based copolymer poly(ethylene glycol)-b-poly(N-(S-(o-nitrobenzyl)-thioethyl) glycine)-co-poly(N-(2-phenylethyl) glycine) (PEG-b-PNSN-co-PNPE) by controlled ring-opening polymerization (ROP). Both $o$-nitrobenzyl group and phenethyl residues have been simultaneously incorporated to tune the property of the system. With UV-irradiation the free thiol groups can be generated by photocleavage of the $o$-nitrobenzyl (NB) groups. The self-assembled morphology transforms from vesicles of a few hundred nanometers to nanospheres of tens nanometers. This process further results in the controllable release of the small molecule by varying the UV-irradiation time. This work provides a facile approach to prepare a series of photoresponsive drug carriers with tunable properties.

Supplementary Materials: The following are available online at http://www.mdpi.com/2073-4360/12/4/798/s1. Scheme S1: Synthetic route of NSN-NCA and NPE-NCA monomer; Figures S1-S3: ${ }^{1} \mathrm{H}$ NMR spectra of NSN-NCA, NPE-NCA, and PEG- $b$-PNSN ${ }_{m}-c o-P N P E_{n}$; Figure S4: GPC traces of the triblock random copolymers; Figure S5: DSC thermograms of PEG- $b$-PNSN ${ }_{9}-c o-\mathrm{PNPE}_{34}$ with non-irradiation (a) and with $10 \mathrm{~h}$ irradiation (b); Figure S6: ${ }^{1} \mathrm{H}$ NMR spectra PEG- $b$-PNSN ${ }_{\mathrm{m}}-\mathrm{co}-\mathrm{PNPE}_{\mathrm{n}}$ with different UV-irradiation time; Figure S7: The TEM images 
of PEG- $b-\mathrm{PNSN}_{\mathrm{m}}-\mathrm{co}-\mathrm{PNPE}_{\mathrm{n}}$ with non-irradiation and with $10 \mathrm{~h}$ irradiation in aqueous solution; Figure S8: The diameter of PEG- $b$-PNSN $\mathrm{m}_{\mathrm{m}}-\mathrm{co}-\mathrm{PNPE}_{\mathrm{n}}$ with non-irradiation and with $10 \mathrm{~h}$ irradiation in aqueous solution determined by DLS.

Author Contributions: The manuscript was written through contributions of all authors. J.S. and X.Y. designed research; X.Y. and J.S. performed research; J.S., X.Y., and Z.W. analyzed data; and J.S., and X.Y. wrote the paper. All authors have given approval to the final version of the manuscript.

Funding: This work was supported by National Natural Science Foundation of China (51722302 and 21674054) and Taishan Scholars Program.

Conflicts of Interest: The authors declare no conflict of interest.

\section{References}

1. Li, Y.M.; Qian, Y.F.; Liu, T.; Zhang, G.Y.; Liu, S.Y. Light-Triggered Concomitant Enhancement of Magnetic Resonance Imaging Contrast Performance and Drug Release Rate of Functionalized Amphiphilic Diblock Copolymer Micelles. Biomacromolecules 2012, 13, 3877-3886. [CrossRef] [PubMed]

2. Liu, G.; Zhou, L.; Guan, Y.; Su, Y.; Dong, C.M. Multi-responsive polypeptidosome: Characterization, morphology transformation, and triggered drug delivery. Macromol. Rapid Commun. 2014, 35, 1673-1678. [CrossRef] [PubMed]

3. Liu, G.; Dong, C.M. Photoresponsive poly(S-(o-nitrobenzyl)-L-cysteine)-b-PEO from a L-cysteine $\mathrm{N}$-carboxyanhydride monomer: Synthesis, self-assembly, and phototriggered drug release. Biomacromolecules 2012, 13, 1573-1583. [CrossRef] [PubMed]

4. Yan, L.S.; Yang, L.X.; He, H.Y.; Hu, X.L.; Xie, Z.G.; Huang, Y.B.; Jing, X.B. Photo-cross-linked mPEG-poly(gamma-cinnamyl-L-glutamate) micelles as stable drug carriers. Polym. Chem. 2012, 3, 1300-1307. [CrossRef]

5. Jain, A.K.; Gund, M.G.; Desai, D.C.; Borhade, N.; Senthilkumar, S.P.; Dhiman, M.; Mangu, N.K.; Mali, S.V.; Dubash, N.P.; Halder, S.; et al. Mutual prodrugs containing bio-cleavable and drug releasable disulfide linkers. Bioorg. Chem. 2013, 49, 40-48. [CrossRef]

6. Liu, G.; Liu, N.; Zhou, L.; Su, Y.; Dong, C.-M. NIR-responsive polypeptide copolymer upconversion composite nanoparticles for triggered drug release and enhanced cytotoxicity. Polym. Chem. 2015, 6, 4030-4039. [CrossRef]

7. Riber, C.F.; Smith, A.A.; Zelikin, A.N. Self-Immolative Linkers Literally Bridge Disulfide Chemistry and the Realm of Thiol-Free Drugs. Adv. Healthc. Mater. 2015, 4, 1887-1890. [CrossRef]

8. Xuan, J.; Boissiere, O.; Zhao, Y.; Yan, B.; Tremblay, L.; Lacelle, S.; Xia, H.S.; Zhao, Y. Ultrasound-Responsive Block Copolymer Micelles Based on a New Amplification Mechanism. Langmuir 2012, 28, 16463-16468. [CrossRef]

9. Il'ichev, Y.V.; Schworer, M.A.; Wirz, J. Photochemical reaction mechanisms of 2-nitrobenzyl compounds: Methyl ethers and caged ATP. J. Am. Chem. Soc. 2004, 126, 4581-4595. [CrossRef]

10. Gaplovsky, M.; Il'ichev, Y.V.; Kamdzhilov, Y.; Kombarova, S.V.; Mac, M.; Schworer, M.A.; Wirz, J. Photochemical reaction mechanisms of 2-nitrobenzyl compounds: 2-nitrobenzyl alcohols form 2-nitroso hydrates by dual proton transfer. Photochem. Photobiol. Sci. 2005, 4, 33-42. [CrossRef]

11. Huo, H.H.; Ma, X.Y.; Dong, Y.Q.; Qu, F.J. Light/temperature dual-responsive ABC miktoarm star terpolymer micelles for controlled release. Eur. Polym. J. 2017, 87, 331-343. [CrossRef]

12. Zhu, K.N.; Liu, G.H.; Zhang, G.Y.; Hu, J.M.; Liu, S.Y. Engineering Cross-Linkable Plasmonic Vesicles for Synergistic Chemo-Photothermal Therapy Using Orthogonal Light Irradiation. Macromolecules 2018, 51, 8530-8538. [CrossRef]

13. Li, P.; Dong, C.M. Phototriggered Ring-Opening Polymerization of a Photocaged 1-Lysine N-Carboxyanhydride to Synthesize Hyperbranched and Linear Polypeptides. ACS Macro Lett. 2017, 6, 292-297. [CrossRef]

14. Wu, X.; Zhou, L.; Su, Y.; Dong, C.M. Plasmonic, Targeted, and Dual Drugs-Loaded Polypeptide Composite Nanoparticles for Synergistic Cocktail Chemotherapy with Photothermal Therapy. Biomacromolecules 2016, 17, 2489-2501. [CrossRef] [PubMed] 
15. Li, P.; Zhang, J.; Dong, C.M. Photosensitive poly(o-nitrobenzyloxycarbonyl-1-lysine)-b-PEO polypeptide copolymers: Synthesis, multiple self-assembly behaviors, and the photo/pH-thermo-sensitive hydrogels. Polym. Chem. 2017, 8, 7033-7043. [CrossRef]

16. Gao, Y.; Dong, C.M. Quadruple thermo-photo-redox-responsive random copolypeptide nanogel and hydrogel. Chin. Chem. Lett. 2018, 29, 927-930. [CrossRef]

17. Murphy, R.D.; Kimmins, S.; Hibbitts, A.J.; Heise, A. 3D-extrusion printing of stable constructs composed of photoresponsive polypeptide hydrogels. Polym. Chem. 2019, 10, 4675-4682. [CrossRef]

18. Fairbanks, B.D.; Singh, S.P.; Bowman, C.N.; Anseth, K.S. Photodegradable, Photoadaptable Hydrogels via Radical-Mediated Disulfide Fragmentation Reaction. Macromolecules 2011, 44, 2444-2450. [CrossRef]

19. Soorkia, S.; Dehon, C.; Kumar, S.S.; Pedrazzani, M.; Frantzen, E.; Lucas, B.; Barat, M.; Fayeton, J.A.; Jouvet, C. UV Photofragmentation Dynamics of Protonated Cystine: Disulfide Bond Rupture. J. Phys. Chem. Lett. 2014, 5, 1110-1116. [CrossRef]

20. Zuckermann, R.N. Peptoid origins. Biopolymers 2011, 96, 545-555. [CrossRef]

21. Sun, J.; Zuckermann, R.N. Peptoid polymers: A highly designable bioinspired material. ACS Nano 2013, 7 , 4715-4732. [CrossRef] [PubMed]

22. Chan, B.A.; Xuan, S.T.; Li, A.; Simpson, J.M.; Sternhagen, G.L.; Yu, T.Y.; Darvish, O.A.; Jiang, N.S.; Zhang, D.H. Polypeptoid polymers: Synthesis, characterization, and properties. Biopolymers 2018, 109, 25. [CrossRef] [PubMed]

23. Sun, J.; Li, Z. Peptoid applications in biomedicine and nanotechnology. Pept. Appl. Biomed. Biotechnol. Bioeng. 2018, 183-213. [CrossRef]

24. Fu, X.; Tian, J.; Li, Z.; Sun, J.; Li, Z. Dual-responsive pegylated polypeptoids with tunable cloud point temperatures. Biopolymers 2018, 110, e23243. [CrossRef]

25. Tian, J.; Sun, J.; Li, Z. Biomimetic pegylated polypeptoids with thermoresponsive properties. Polymer 2018, 138, 132-138. [CrossRef]

26. Xing, C.; Shi, Z.; Tian, J.; Sun, J.; Li, Z. Charge-Determined LCST/UCST Behavior in Ionic Polypeptoids. Biomacromolecules 2018, 19, 2109-2116. [CrossRef]

27. Ji, S.; Xu, L.; Fu, X.; Sun, J.; Li, Z. Light- and Metal Ion-Induced Self-Assembly and Reassembly Based on Block Copolymers Containing a Photoresponsive Polypeptide Segment. Macromolecules 2019, 52, 4686-4693. [CrossRef]

28. Wei, J.; Sun, J.; Yang, X.; Ji, S.; Wei, Y.; Li, Z. Self-crosslinking assemblies with tunable nanostructures from photoresponsive polypeptoid-based block copolymers. Polym. Chem. 2020, 11, 337-343. [CrossRef]

29. Wei, Y.; Tian, J.; Zhang, Z.; Zhu, C.; Sun, J.; Li, Z. Supramolecular Nanosheets Assembled from Poly(ethylene glycol)-b-poly(N-(2-phenylethyl)glycine) Diblock Copolymer Containing Crystallizable Hydrophobic Polypeptoid: Crystallization Driven Assembly Transition from Filaments to Nanosheets. Macromolecules 2019, 52, 1546-1556. [CrossRef]

(C) 2020 by the authors. Licensee MDPI, Basel, Switzerland. This article is an open access article distributed under the terms and conditions of the Creative Commons Attribution (CC BY) license (http://creativecommons.org/licenses/by/4.0/). 